\title{
Measurement invariance of the short Warwick-Edinburgh Mental Wellbeing Scale and latent mean differences (SWEMWBS) in young people by current care status
}

\author{
Rebecca Anthony $^{1,2}$ (D) $\cdot$ Graham Moore $^{1} \cdot$ Nicholas Page $^{1} \cdot$ Gillian Hewitt $^{1,2} \cdot$ Simon Murphy $^{1,2} \cdot$ G. J. Melendez-Torres $^{3}$
}

Accepted: 24 May 2021 / Published online: 28 May 2021

(c) The Author(s) 2021

\begin{abstract}
Purpose Studying mental wellbeing requires the use of reliable, valid, and practical assessment tools, such as the Short version of the Warwick-Edinburgh Mental Wellbeing Scale (SWEMWBS). Research on the mental wellbeing of children in care is sparse. The current study aims to: (1) examine the unidimensionality of SWEMWBS; (2) assess measurement invariance of SWEMWBS across children and young people in care compared to their peers not in care; and (3) investigate the latent factor mean differences between care status groups.

Methods We used data from the 2017 School Health Research Network Student Health and Wellbeing (SHW) survey, completed by 103,971 students in years 7 to 11 from 193 secondary schools in Wales. The final data include a total of 2,795 participants ( $46 \%$ boys), which includes all children in care and a sub-sample of children not in care who completed the SWEMWBS scale fully and answered questions about their living situation.

Results Confirmatory factor analysis supported the unidimensionality of SWEMWBS. The SWEMWBS is invariant across groups of young people in foster, residential and kinship care compared to children and young people not in care at configural, metric and scalar levels. Findings from latent mean comparisons showed that young people in care reported lower mental wellbeing than their peers, with those in residential care reporting the lowest scores.

Conclusions Findings suggest that SWEMWBS is a valid scale for measuring differences in mental wellbeing for young people in care similar to the population.
\end{abstract}

Keywords Mental wellbeing $\cdot$ Adolescents $\cdot$ Looked after $\cdot$ Care $\cdot$ Measure $\cdot$ Psychometric properties

\section{Background}

Mental wellbeing has emerged as an important construct in population health, described as a fundamental human right and essential for a sustainable and functional society [1].

Rebecca Anthony

AnthonyRE@cardiff.ac.uk

Graham Moore

Mooreg@cardiff.ac.uk

Nicholas Page

PageN2@cardiff.ac.uk

Gillian Hewitt

HewittG@cardiff.ac.uk

Simon Murphy

Murphys7@cardiff.ac.uk

G. J. Melendez-Torres

G.j.melendez-torres@exeter.ac.uk
Mental wellbeing has been defined as 'a state of wellbeing in which every individual realises his or her own potential, can cope with the normal stresses of life, can work productively and fruitfully, and is able to make a contribution to her or his community' [2], encompassing concepts such as resilience,

1 Centre for Development, Evaluation, Complexity, and Implementation in Public Health Improvement (DECIPHer), School of Social Sciences, Cardiff University, 1-3 Museum Place, Cardiff CF10 3BD, UK

2 Wolfson Centre for Young People's Mental Health, Cardiff University, Cardiff, UK

3 College of Medicine and Health, South Cloisters, University of Exeter, St Luke's Campus, Heavitree Road, Exeter EX1 2LU, UK 
self-efficacy and optimism [3]. As opposed to mental illness, which is either prevented or treated, mental wellbeing can only be promoted [4], and promotion has been shown to relate to improved health and longevity in adults [5]. Despite this, little information is available on the prevalence or social patterning of mental wellbeing in young people [3], particularly compared to the extensive data on mental illness [6].

In the United Kingdom (UK), local authority care includes the provision of accommodation for children and young people who are unable to live with their parents. There are a variety of reasons for young people to enter care, with approximately two thirds entering care due to abuse and neglect [7]. As of March 2019, 6846 young people in Wales were in the care of their local authority, the majority (71\%) accommodated in foster care placements [7]. While most young people in care in the UK report their experiences to be good [8], and report satisfaction with their life [9], there is clear evidence that those who have experienced care do not fare as well as the general population in relation to their physical health, cognitive and language skills [10], and mental health [11-13], which in turn can impact their development and journey to adulthood [14-16]. Studies have begun to investigate subjective wellbeing of children and young people in foster care in the UK [9], and foster and residential care internationally [17-19]. These studies have consistently identified lower levels of subjective wellbeing of those in care compared to their peers not in care, with those in residential care demonstrating the lowest levels of wellbeing.

Studying mental wellbeing requires the use of reliable measurement tools. The Warwick-Edinburgh Mental Wellbeing Scale (WEMWBS) was developed in 2007 [20] and is one of the most widely used measures of mental wellbeing [21]. It contains 14-items covering both psychological functioning and subjective wellbeing facets of mental wellbeing. A brief seven-item version (SWEMWBS) was subsequently developed using the Rasch measurement model which had preferable psychometric properties to the full version, though it is focussed more on functioning than subjective aspects of mental wellbeing [22]. While measures of subjective wellbeing have been developed for young people in foster care, including specific care-related aspects such as birth parent contact [23], a brief measure of mental wellbeing may be of particular use in population research where practical constraints often restrict the scope for detailed surveys.

It is often assumed that scores represent the same level of the construct for members of different groups. However, the nature and magnitude of relationships between items and a latent construct may differ across groups, meaning that comparisons between groups cannot be meaningfully made unless the measure is capturing the same thing in each sub-group [24-26]. Thus, if we want to know if policies and interventions are working as well for children in care as for the rest of the population, we need to be able to measure this equally well in both groups. Testing for invariance of measures makes it possible to verify whether the members of different groups or cultures ascribe the same meanings to the items of a questionnaire [27], which is critical for informing both practice and research [28]. Most studies examining the psychometric properties of SWEMWBS have been undertaken in adults [22, 29, 30]; the few studies conducted with adolescents have found acceptable measurement invariance properties by age and gender [31,32], and demonstrate good external construct validity [32].

\section{The present study}

The aims of the present study were to: (1) Confirm the unidimensionality of SWEMWBS; (2) assess measure invariance of SWEMWBS across children and young people in care compared to their peers not in care; and (3) undertake comparison of mean differences in mental wellbeing across those groups. While other specific mental health measures, such as the Strengths and Difficulties Questionnaire [33], have been examined across groups of care-experienced children and young people, to the best of our knowledge no study has yet examined the equivalence of SWEMWBS across these groups.

\section{Method}

We used a population sample $(N=2795)$ of young people in Wales to examine the measurement invariance properties of SWEMWBS across groups of young people currently in the care of the local authority (i.e. foster, residential or kinship care placements) compared to their peers of a similar age not in care.

\section{Study sample}

We used data from the 2017 School Health Research Network Student Health and Wellbeing (SHW) survey, reported in detail elsewhere $[32,34,35]$. The survey was completed by $N=103,971$ students in years 7 to 11 , representing 193 secondary schools in Wales. The SHW survey is an online, closed response, self-completion survey, available in English and Welsh. The survey measures self-reported health behaviours among school students aged 11-16 years (i.e. years 7 to 11 of the British secondary school system), and includes questions from the current round of the international HBSC survey [36] alongside additional questions reflecting current policy, practice and research priorities in Wales. Students completed the survey during school hours between September and December of the autumn term of the 2017-2018 school year and could opt out of the survey. A 
total of $n=79,297$ completed the scale fully and answered questions about their living situation. At the time of the survey, $n=77,588$ (97.84\%) were not currently living in care, $n=513(0.65 \%)$ currently lived-in local authority foster care, $n=126(0.16 \%)$ currently lived-in local authority residential care and $n=1,070(1.33 \%)$ were in kinship care. Due to simulation studies showing that severely unbalanced group size conditions are more likely to reduce power and can mask violations of invariance $[37,38]$, rather than include all children not living in local authority care a sub-sample of children and young people not in care were randomly selected using the 'sample' command in Stata to be similar in size to the largest care group (approximately $1.4 \%$ of the cases) $(n=1086)$. Thus, the final sample included $N=2,795$ children and young people.

\section{Measures}

\section{Care status}

All respondents were asked the following question to assess their current living arrangements. "All families are different (for example, not everyone lives with both their parents; sometimes people live with just one parent, they have two homes, or live with two families) and we would like to know about yours. Please answer this question for the home where you live all or most of the time and tick the ADULTS who live there". The options included mother, father, mothers partner, fathers partner, grandparent(s), aunt(s)/uncle(s), adult brother(s)/sister(s), foster parents, residential care or a children's home, independently (on my own or with friends or my partner), and someone or somewhere else. Responses were then categorised into 'not in care', 'foster care', 'residential care' or 'kinship care'. Observations where students either responded 'I do not want to answer', left a question blank or answered yes to eight or more possible living arrangement options (considered implausible) were set to missing.

\section{SWEMWBS}

All students were presented with the seven questions comprising SWEMWBS [20]: 'I've been feeling optimistic about the future', 'I've been feeling useful', 'I've been feeling relaxed', 'I've been dealing with problems well', 'I've been thinking clearly', 'I've been feeling close to other people', and 'I've been able to make up my own mind about things' alongside the following question: 'Below are some statements about feelings and thoughts. Please select the option that best describes your experience of each over the last 2 weeks'. For each question, students could select one of five frequency options: 'none of the time', 'rarely', 'some of the time', 'often' and 'all of the time'.

\section{Statistical analysis}

Stata v.14 [39] was used to conduct descriptive statistics (i.e. minimum, maximum, mean, and standard deviation) for each sample across all seven items of the SWEWMBS scale and to estimate polychoric correlation matrices for the whole sample and by care status.

Following this, lavaan 0.6-3 [40] and semTools 0.5-1 [41] R packages were used to conduct confirmatory factor analysis (CFA) to test the unidimensionality of the SWEMWBS scale and measurement invariance tests. Categorical confirmatory factor analysis was performed using diagonally weighted (DWLS) estimators suitable for ordinally scaled responses [42]. All standardised factor loadings within this single factor should be above 0.5 and statistically significant [43] to support the unidimensionality. Model fit was assessed using Chi-square $\left(\chi^{2}\right)$ and its degrees of freedom (test values associated with $p>0.05$ ), the Comparative-ofFit Index (CFI; values $\geq 0.90$ ), Tucker-Lewis Index (TLI; values $\geq 0.90$ ), Root Mean Square Error of Approximation (RMSEA; values close to 0.06 ) and its $95 \%$ confidence interval (CI), and Standardised Root Mean Square Residual (SRMR; values $\leq 0.08$ ) as advised [44].

In accordance with Jöreskog's strategy [45], we used a multiple-groups structural equation model with successively greater constraints that tested for configural invariance and scalar invariance [46, 47], following the recommended procedure and syntax described by Svetina et al. (2020) [48]. Because of the ordinal nature of the individual items, we again used a diagonally weighted least squares estimator with a scale-shifted test statistic. We first estimated a baseline (configural) where thresholds and loadings are estimated freely using delta parameterization. Next, we estimated a model where the thresholds where constrained to be equal, and finally we estimated a model where both the thresholds and loadings (scalar) are constrained to be equal. The scalar invariance test is a strong invariance test and the establishment of this test is required before the latent means can be compared across groups.

The performance of fit indices for invariance tests with categorical or ordinal data has not been adequately studied [49] and is still a developing area, particularly for ordinal data [50] where there remains a lack of agreement among scholars as to which recommendation to adopt [48]. However, based on recommendations and the size of our sample, we did not use traditional $\chi^{2}$ tests for invariance. Instead, we used the comparative fit index (CFI) and the root mean squared error of approximation (RMSEA). The CFI has previously been shown to be an appropriate index of measurement invariance, with decrements of greater than -0.01 in successive models suggesting that measurement variance is not appropriate [51]. In addition, emerging evidence shows promise for the RMSEA as an information criterion, where 
the lowest value indicates the model with the best trade-off between fit and complexity [52].

Following measurement invariance testing, we compared latent mean differences between care status group. Specifically, a full scalar invariance model was used as the baseline. To compare differences in latent means between groups, we constrained the 'not in care' latent mean to 0 and the latent means of the foster care, kinship care and residential care groups were free to estimate [53].

\section{Results}

Descriptive statistics for the sample can be found in Table 1. The response category proportions as well as item means, and standard deviations (data treated as continuous) can be found in Table 2. Across all items, it is worth noting that most children and young people positively rate items assessing their mental wellbeing. Visual examination of polychoric correlation matrices showed significant interitems
Table 1 Sample descriptive statistics (N/\%)

\begin{tabular}{|c|c|c|c|c|c|}
\hline Variable & $\begin{array}{l}\text { Total sample } \\
(N=2795)\end{array}$ & $\begin{array}{l}\text { Not in care } \\
(N=1086)\end{array}$ & $\begin{array}{l}\text { Foster care } \\
(N=513)\end{array}$ & $\begin{array}{l}\text { Residential } \\
\text { care }(N=126)\end{array}$ & $\begin{array}{l}\text { Kin- } \\
\text { ship care } \\
(N=1070)\end{array}$ \\
\hline \multicolumn{6}{|l|}{ Gender } \\
\hline Male & $1273(46)$ & $506(47)$ & $234(46)$ & $59(47)$ & $474(44)$ \\
\hline Female & $1464(52)$ & $571(53)$ & $263(51)$ & $53(42)$ & $577(54)$ \\
\hline Prefer not to say & $58(2)$ & $9(1)$ & $16(3)$ & $14(11)$ & $19(2)$ \\
\hline \multicolumn{6}{|l|}{ School year } \\
\hline Year 7 & $513(18)$ & $199(18)$ & $106(21)$ & $25(20)$ & $183(17)$ \\
\hline Year 8 & $550(20)$ & $213(20)$ & $103(20)$ & $25(20)$ & $209(20)$ \\
\hline Year 9 & $617(22)$ & $242(22)$ & $110(21)$ & $28(22)$ & $237(22)$ \\
\hline Year 10 & $570(20)$ & $210(19)$ & $108(21)$ & $33(26)$ & $219(20)$ \\
\hline Year 11 & $545(20)$ & $222(20)$ & $86(17)$ & $15(12)$ & $222(21)$ \\
\hline \multicolumn{6}{|l|}{ Family affluence } \\
\hline Low & $1022(39)$ & $359(34)$ & $150(32)$ & $38(35)$ & $475(46)$ \\
\hline Medium & $822(31)$ & $325(31)$ & $143(30)$ & $36(33)$ & $318(31)$ \\
\hline High & $810(31)$ & $365(35)$ & $179(38)$ & $34(31)$ & $232(23)$ \\
\hline \multicolumn{6}{|l|}{ Ethnicity } \\
\hline White British & $2307(85)$ & $915(86)$ & $412(83)$ & $72(60)$ & $908(87)$ \\
\hline White non-British & $158(6)$ & $46(4)$ & $45(9)$ & $16(13)$ & $51(5)$ \\
\hline Black and Minority Ethnic & $256(9)$ & $98(9)$ & $42(8)$ & $33(27)$ & $83(8)$ \\
\hline \multicolumn{6}{|l|}{ Language } \\
\hline English & $2691(96)$ & $1029(95)$ & $499(97)$ & $117(93)$ & $1046(98)$ \\
\hline Welsh & $104(4)$ & $57(5)$ & $14(3)$ & $9(7)$ & $24(2)$ \\
\hline
\end{tabular}

Table 2 Item descriptive statistics

\begin{tabular}{|c|c|c|c|c|c|c|}
\hline SWEMWBS items* & $\begin{array}{l}\text { 'none of the } \\
\text { time' }(\mathrm{N} / \%)\end{array}$ & 'rarely' (N/\%) & $\begin{array}{l}\text { 'some of the } \\
\text { time' (N/\%) }\end{array}$ & ‘often’ (N/\%) & 'all of the time' (N/\%) & Mean \pm SD \\
\hline $\begin{array}{l}\text { Item } 1 \text { "I've been feeling optimistic about the } \\
\text { future" }\end{array}$ & $379(13.56)$ & $562(20.11)$ & $830(29.70)$ & $678(24.26)$ & $346(12.38)$ & $3.02 \pm 1.22$ \\
\hline Item 2 "I've been feeling useful" & $320(11.45)$ & 579 (20.72) & 964 (34.49) & 667 (23.86) & $265(9.48)$ & $2.99 \pm 1.13$ \\
\hline Item 3 "I've been feeling relaxed" & $249(8.91)$ & $510(18.25)$ & $803(28.73)$ & $806(28.84)$ & $427(15.28)$ & $3.23 \pm 1.18$ \\
\hline Item 4 "I've been dealing with problems well" & $323(11.56)$ & $534(19.11)$ & $804(28.77)$ & $751(26.87)$ & $383(13.70)$ & $3.12 \pm 1.21$ \\
\hline Item 5 "I've been thinking clearly" & $247(8.84)$ & $492(17.60)$ & $832(29.77)$ & $769(27.51)$ & $455(16.28)$ & $3.25 \pm 1.18$ \\
\hline Item 6 "I've been feeling close to other people" & $236(8.44)$ & $419(14.99)$ & $667(23.86)$ & $783(28.01)$ & $690(24.69)$ & $3.46 \pm 1.24$ \\
\hline $\begin{array}{l}\text { Item } 7 \text { "I've been able to make up my own } \\
\text { mind about things" }\end{array}$ & $171(6.12)$ & $300(10.73)$ & $582(20.82)$ & $841(30.09)$ & $901(32.24)$ & $3.72 \pm 1.20$ \\
\hline
\end{tabular}

*WEMWBS is protected by copyright. Those wishing to use WEMWBS can obtain a licence to do so. Please go to https://warwick.ac.uk/wemwbs/using for information on the type of licence you will require and details on how to apply 
correlation coefficients, ranging from 0.30 to 0.56 for the full sample (see Online Table S1). The matrices indicated increasing intercorrelation in the different care-experienced groups, with the strongest intercorrelations within the residential care group (ranging from 0.61 to 0.77 ). The scale demonstrated good internal consistency reliability across all groups ( $\alpha=0.84$ in full sample, $\alpha=0.82$ 'not in care' group; $\alpha=0.86$ in Foster Care, $\alpha=0.90$ in Residential Care and $\alpha=0.81$ 'kinship care group).

\section{Factorial structure}

Categorical confirmatory factorial analysis was used to test a unidimensional model in which the scores obtained for the 7 items of the scale all contribute to the evaluation of children and young people's mental wellbeing. Considering the sensitivity of the chi-square statistic to sample size [54], we assessed a number of additional indices. Model fit was assessed to be adequate as despite the significant Chisquare all other indices showed excellent fit $\left(\chi^{2}(\mathrm{df}=14)=1\right.$ 90.75, $p<0.001 ; \mathrm{CFI}=0.988 ; \mathrm{TLI}=0.982 ; \mathrm{RMSEA}=0.067$ [0.059-0.0.076]; SRMR =0.024). Furthermore, all standardised factor loadings were statistically significant $(p<0.001)$ and ranged from 0.548 to 0.814 (item $1=0.548$; item $2=0.703$; item $3=0.701$; item $4=0.743$; item $5=0.814$; item $6=0.657$; item $7=0.718$ ), higher than the threshold of 0.5 [43]. See Online Table S2 for item factor loadings.

\section{Measurement invariance}

Having verified unidimensionality of the SWEMWBS, we estimated a one-factor configural invariance model. Results from each of the successively stricter invariance tests are reported in Table 3. Configural invariance (baseline model) provided an acceptable fit to the data, (CFI 0.984, TLI 0.958, RMSEA 0.073, $p<0.001$ ), meaning the constructs had similar patterns of free and fixed loadings across groups [46]. Metric invariance was subsequently tested whereby factor variances remained freely estimated but factor loadings were held invariant. Findings showed that the model fit the data well and there was no change in CFI (0.000) and a reduction in RMSEA (-0.017), thus the items therefore loaded onto factors similarly across groups [46]. Scalar invariance was then tested whereby indicator thresholds were now also held invariant, fit indices suggested acceptable fit with this constraint as indicated by little change to CFI $(0.003)$ and a reduction in RMSEA (-0.010).

\section{Latent mean differences in mental wellbeing}

Based on the establishment of scalar invariance across care status groups, latent mean comparisons can be made between care status groups. The NIC (not in care) group served as the reference group. Findings showed that young people currently in all types of care reported significantly lower mental wellbeing scores than those not in care (kinship care: $E_{\mathrm{st}}=-0.364 \pm 0.051, p<0.001$; foster care: $\left.E_{\mathrm{st}}=-0.319 \pm 0.075, p<0.001\right)$, and those in residential care reported the lowest levels of mental wellbeing; $\left(E_{\mathrm{st}}=-0.882 \pm 0.192, p<0.001\right)$.

\section{Discussion}

In this study, we analysed the short version of the WEMWBS in a population-based sample of school-aged students to explore measurement invariance and latent mean differences between young people currently in care (foster, kinship and residential settings) and those not currently in the care of local authority. The 1-factor CFA test showed that the SWEMWBS exhibited satisfactory model fit and demonstrated unidimensionality, thus this short single-factor instrument may be useful in reducing respondent burden in future studies. The current study established configural, metric and scalar invariances across care status groups, suggesting that differences in SWEMWBS scores between care status groups can be attributed to differences in the underlying latent trait rather than to the measure itself. Researchers employing SWEMWBS in future studies can compare the mental wellbeing scores meaningfully across those in different types of local authority care compared to their peers not in care.

Our findings revealed that young people in all types of care reported significantly lower mental wellbeing scores than their peers of the same age not in care. This result is consistent with findings from previous studies testing wellbeing scores using traditional methods [17-19]. Research shows that developmentally specific factors including
Table 3 Measurement invariance tests of SWEMWBS across care status

\begin{tabular}{lllll}
\hline Model constraints & CFI scaled & RMSEA scaled (90\% CI) & CFI & RMSEA \\
\hline Configural & 0.984 & $0.073(0.068-0.082)$ & - & - \\
Loadings & 0.984 & $0.056(0.049-0.063)$ & No change & -0.017 \\
Loadings, thresholds & 0.987 & $0.046(0.040-0.053)$ & 0.003 & -0.010 \\
Additive $^{\mathrm{a}}$ & & & 0.003 & -0.027 \\
\hline
\end{tabular}

${ }^{a}$ Additive change from baseline 
parents' availability and wellbeing, family relationships and interactions, quality of care, and supportive learning environments are critical for children's wellbeing [55]. Thus, given that the majority of children entered care due to abuse or neglect [7] and the strong evidence base showing the long-lasting impact of early trauma and adversity [56-59] we suggest that the lower mental wellbeing of those in care may be due to early negative experiences prior to or during care; however, we do not have the data available to test this assumption. Given that the purpose of the care system is to address these factors by: protecting children from further harm, addressing a child's need for good parenting, and enabling them to recover from traumatic experiences [9], further work to promote mental wellbeing of young people in care is needed. A scoping review [6] highlighted a number of interventions which may be beneficial in improving children and young people's wellbeing. The review also highlighted a decreasing emphasis on wellbeing as children grew into teens or young adults, with more interventions available for this age group which intervene in the development of mental illness rather than promoting wellbeing.

Our analysis has several strengths, but also several limitations. Our large-scale nationally representative sample provides evidence of the utility of SWEMWBS for measuring mental wellbeing among young people in care in the UK. A limitation of this study is the lack of testing for invariance across other categories, such as gender, family affluence and ethnicity. Previous studies have shown that SWEMWBS demonstrates strong measurement invariance across sex and age differences in adults [60] and a further study showed measurement invariance across the full age range of secondary school students [32]; however, as previous research has shown that these factors are all connected to strong structural inequities [61], future work should address this limitation by testing for invariance across family affluence and ethnicity. Furthermore, it is possible that results from Wales may not generalise internationally, though evidence of the psychometric properties of SWEMWBS in adults is consistent across multiple cultures [62]. Self-reported data may have been biased by standard limitations (e.g. memory recall biases, social desirability, etc.). While the living situation question enabled us to identify that the 'kinship care' group are living with family other than their parents we cannot be sure if they are subject to a formal care order. As the SHW survey is only completed by young people in mainstream schooling, the views of children not in mainstream school are not included, this is particularly significant given that approximately $40 \%$ of children in care attend non-mainstream schools such as special schools, pupil referral units and alternative provisions [63].

\section{Conclusions}

This study adds to the growing evidence that SWEMWBS is appropriate for measuring mental wellbeing in young people in care. Additionally, the wellbeing of young people in foster, kinship and residential placements was significantly lower than their peers not in care, highlighting the need for interventions to promote mental wellbeing in this group.

Supplementary Information The online version contains supplementary material available at https://doi.org/10.1007/s11136-021-02896-0.

Acknowledgements We thank Chris Roberts within the Social Research and Information Division Welsh Government for his support, and all school staff and students who completed the survey

Author contributions RA and GJM conceived the work. RA analysed and interpreted the data with support from GJM and NP. RA drafted the manuscript and all authors contributed to the revisions of the manuscript and approved the final draft for submission.

Funding This work was supported by The Centre for Development, Evaluation, Complexity and Implementation in Public Health Improvement (DECIPHer) funded by Welsh Government through Health and Care Research Wales and the previous Centre for the Development and Evaluation of Complex Interventions for Public Health Improvement (DECIPHer), a UKCRC Public Health Research Centre of Excellence. Joint funding [Grant Number MR/KO232331/1] from the British Heart Foundation, Cancer Research UK, Economic and Social Research Council, Medical Research Council, the Welsh Government and the Wellcome Trust, under the auspices of the UK Clinical Research Collaboration, is gratefully acknowledged. The Wolfson Centre for Young People's Mental Health was established with support from The Wolfson Foundation.

Data availability (Data transparency). Data underpinning this analysis is available upon reasonable request to the School Health Research Network and completion of the necessary data application documentation.

Code availability (software application or custom code) Code is available on request.

\section{Disclosures}

Conflict of interest The authors declare that they have no conflict of interest.

Ethical approval Ethical approval for the 2017 SHW survey was obtained from Cardiff University's School of Social Sciences Research Ethics Committee, and the research has therefore been performed in accordance with the ethical standards laid down in the 1964 Declaration of Helsinki and its later amendments. Students could opt out of the survey.

Open Access This article is licensed under a Creative Commons Attribution 4.0 International License, which permits use, sharing, adaptation, distribution and reproduction in any medium or format, as long as you give appropriate credit to the original author(s) and the source, provide a link to the Creative Commons licence, and indicate if changes were made. The images or other third party material in this article are 
included in the article's Creative Commons licence, unless indicated otherwise in a credit line to the material. If material is not included in the article's Creative Commons licence and your intended use is not permitted by statutory regulation or exceeds the permitted use, you will need to obtain permission directly from the copyright holder. To view a copy of this licence, visit http://creativecommons.org/licenses/by/4.0/.

\section{References}

1. Barry, M., Friedli, L. (2010). The influence of social, demographic and physical factors on positive mental health in children, adults and older people.

2. World Health Organization. (2001). The World Health Report 2001: Mental health: New understanding, new hope; World Health Organization.

3. Barry, M. M. (2009). Addressing the determinants of positive mental health: Concepts, evidence and practice. International Journal of Mental Health Promotion, 11, 4-17.

4. Keyes, C. L. (2002). The mental health continuum: From languishing to flourishing in life. Journal of health and social behavior., 20, 207-222.

5. Diener, E., \& Chan, M. Y. (2011). Happy people live longer: Subjective well-being contributes to health and longevity. Applied Psychology, 3, 1-43.

6. Welsh, J., Strazdins, L., Ford, L., Friel, S., O’Rourke, K., Carbone, S., \& Carlon, L. (2015). Promoting equity in the mental wellbeing of children and young people: a scoping review. Health Promotion International, 30, ii36-ii76. https://doi.org/ 10.1093/heapro/dav053

7. Welsh Government. (2019). Experimental Statistics: Children looked after by local authorities, 2018-2019.

8. Biehal, N., Cusworth, L.S., Wade, J., Clarke, S.E. (2014). Keeping children safe: Allegations concerning the abuse or neglect of children in care.

9. Selwyn, J., Wood, M., \& Newman, T. (2017). Looked after children and young people in England: Developing measures of subjective well-being. Child Indicators Research, 10, 363-380.

10. Harden, B. J., \& Whittaker, J. V. (2011). The early home environment and developmental outcomes for young children in the child welfare system. Children and Youth Services Review, 33, 1392-1403.

11. Teyhan, A., Wijedasa, D., \& Macleod, J. (2018). Adult psychosocial outcomes of men and women who were looked-after or adopted as children: Prospective observational study. British Medical Journal Open, 8, e019095.

12. Ford, T., Vostanis, P., Meltzer, H., Goodman, R. (2007). Psychiatric disorder among British children looked after by local authorities: Comparison with children living in private households. The British Journal of Psychiatry, 190, 319-325.

13. Anthony, R. E., Paine, A. L., \& Shelton, K. H. (2019). Adverse childhood experiences of children adopted from care: The importance of adoptive parental warmth for future child adjustment. International Journal of Environmental Research and Public Health, 16, 2212.

14. Wade, J., \& Dixon, J. (2006). Making a home, finding a job: Investigating early housing and employment outcomes for young people leaving care. Child \& Family Social Work, 11, 199-208.

15. Kools, S., Paul, S. M., Norbeck, J. S., \& Robbins, N. R. (2009). Dimensions of health in young people in foster care. International Journal of Adolescent Medicine and Health, 21, 221.
16. Conn, A.-M., Szilagyi, M. A., Jee, S. H., Blumkin, A. K., \& Szilagyi, P. G. (2015). Mental health outcomes among child welfare investigated children: In-home versus out-of-home care. Children and Youth Services Review, 57, 106-111.

17. Llosada-Gistau, J., Casas, F., \& Montserrat, C. (2020). Factors Influencing the subjective well-being of adolescents in out-ofhome care: a mixed method Study. Applied Research in Quality of Life, 15, 835-863.

18. Delgado, P., Carvalho, J. M., Montserrat, C., \& Llosada-Gistau, J. (2020). The subjective well-being of Portuguese children in foster care, residential care and children living with their families: Challenges and implications for a child care system still focused on institutionalization. Child Indicators Research, 13, $67-84$.

19. Schütz, F.F., Cassarino-Perez, L., Córdova, V.E. (2017). Subjective Well-being of children in residential care. In Psychosocial well-being of children and adolescents in Latin America; Springer. pp. 47-70.

20. Tennant, R., Hiller, L., Fishwick, R., Platt, S., Joseph, S., Weich, S., Parkinson, J., Secker, J., \& Stewart-Brown, S. (2007). The Warwick-Edinburgh mental well-being scale (WEMWBS): development and UK validation. Health and Quality of Life Outcomes. https://doi.org/10.1186/1477-7525-5-63

21. Ng Fat, L., Scholes, S., Boniface, S., Mindell, J., \& StewartBrown, S. (2017). Evaluating and establishing national norms for mental wellbeing using the short Warwick-Edinburgh Mental Well-being Scale (SWEMWBS): Findings from the Health Survey for England. Quality of Life Research, 26, 1129-1144. https://doi. org/10.1007/s11136-016-1454-8

22. Stewart-Brown, S., Tennant, A., Tennant, R., Platt, S., Parkinson, J., \& Weich, S. (2009). Internal construct validity of the Warwick-Edinburgh Mental Well-being Scale (WEMWBS): a Rasch analysis using data from the Scottish Health Education Population Survey. Health and Quality of Life Outcomes, 7, 15. https://doi. org/10.1186/1477-7525-7-15

23. Zhang, M.F., Selwyn, J. (2019) The subjective well-being of children and young people in out of home care: Psychometric analyses of the "Your Life, your Care" survey. Child Indicators Research, pp. 1-24.

24. Dimitrov, D. M. (2010). Testing for factorial invariance in the context of construct validation. Measurement and Evaluation in Counseling and Development, 43, 121-149.

25. Muthén, B., \& Asparouhov, T. (2002). Latent variable analysis with categorical outcomes: Multiple-group and growth modeling in Mplus. Mplus Web Notes, 4, 1-22.

26. Raykov, T., Marcoulides, G. A., \& Millsap, R. E. (2013). Factorial invariance in multiple populations: A multiple testing procedure. Educational and Psychological Measurement, 73, 713-727.

27. Svetina, D., Rutkowski, L., \& Rutkowski, D. (2020). Multiplegroup invariance with categorical outcomes using updated guidelines: An illustration using M plus and the lavaan/semtools packages. Structural Equation Modeling, 27, 111-130.

28. Bowen, N. K., \& Masa, R. D. (2015). Conducting measurement invariance tests with ordinal data: A guide for social work researchers. Journal of the Society for Social Work and Research, 6, 229-249.

29. Haver, A., Akerjordet, K., Caputi, P., Furunes, T., \& Magee, C. (2015). Measuring mental well-being: A validation of the Short Warwick-Edinburgh Mental Well-Being Scale in Norwegian and Swedish. Scandinavian Journal of Public Health, 43, 721-727. https://doi.org/10.1177/1403494815588862

30. Smith, O. R. F., Alves, D. E., Knapstad, M., Haug, E., \& Aaro, L. E. (2017). Measuring mental well-being in Norway: Validation of the Warwick-Edinburgh Mental Well-being Scale (WEMWBS). BMC Psychiatry. https://doi.org/10.1186/s12888-017-1343-x 
31. Smith, O. R., Alves, D. E., Knapstad, M., Haug, E., \& Aarø, L. E. (2017). Measuring mental well-being in Norway: Validation of the Warwick-Edinburgh Mental Well-being Scale (WEMWBS). BMC Psychiatry, 17, 182.

32. Melendez-Torres, G., Hewitt, G., Hallingberg, B., Anthony, R., Collishaw, S., Hall, J., Murphy, S., \& Moore, G. (2019). Measurement invariance properties and external construct validity of the short Warwick-Edinburgh mental wellbeing scale in a large national sample of secondary school students in Wales. Health and Quality of Life Outcomes, 17, 139.

33. Ford, T., Vostanis, P., Meltzer, H., \& Goodman, R. (2007). Psychiatric disorder among British children looked after by local authorities: comparison with children living in private households. British Journal of Psychiatry, 190, 319-325. https://doi.org/10. 1192/bjp.bp.106.025023

34. Hewitt, G., Anthony, R., Moore, G., Melendez-Torres, G., Murphy, S. (2019). Student health and wellbeing in wales: Report of the 2017/18 Health Behaviour in School-aged Children Survey and School Health Research Network Student Health and Wellbeing Survey.

35. Murphy, S., Littlecott, H., Hewitt, G., MacDonald, S., Roberts, J., Bishop, J., Roberts, C., Thurston, R., Bishop, A., Moore, L., et al. (2018). A transdisciplinary complex adaptive systems (T-CAS) approach to developing a national school-based culture of prevention for health improvement: the school health research network (SHRN) in wales. Prevention Science. https://doi.org/10.1007/ s11121-018-0969-3

36. Roberts, C., Freeman, J., Samdal, O., Schnohr, C. W., de Looze, M. E., Gabhainn, S. N., Iannotti, R., Rasmussen, M., \& Grp, I. H. S. (2009). The health behaviour in school-aged children (HBSC) study: Methodological developments and current tensions. International Journal of Public Health, 54, 140-150. https://doi.org/ 10.1007/s00038-009-5405-9

37. Yoon, M., \& Lai, M. H. C. (2018). Testing factorial invariance with unbalanced samples. Structural Equation Modeling, 25, 201-213. https://doi.org/10.1080/10705511.2017.1387859

38. Chen, F. F. (2007). Sensitivity of goodness of fit indexes to lack of measurement invariance. Structural Equation Modeling, 14, 464-504. https://doi.org/10.1080/10705510701301834

39. Statacorp. (2013).

40. Rosseel, Y. (2012). lavaan: An R package for structural equation modeling. Journal of Statistical Software, 48, 1-36.

41. Jorgensen, T.D., Pornprasertmanit, S., Schoemann, A. M., \& Rosseel, Y. (2021). semTools: Useful tools for structural equation modeling. R package version 0.5-4.

42. Forero, C. G., Maydeu-Olivares, A., \& Gallardo-Pujol, D. (2009). Factor analysis with ordinal indicators: A monte carlo study comparing DWLS and ULS estimation. Structural Equation Modeling, 16, 625-641. https://doi.org/10.1080/10705510903203573

43. Hair, J. F., Black, W. C., Babin, B. J., Anderson, R. E., \& Tatham, R. L. (1998). Multivariate data analysis. Saddle River: Prentice Hall Upper.

44. Hu, L. T., \& Bentler, P. M. (1999). Cutoff criteria for fit indexes in covariance structure analysis: Conventional criteria versus new alternatives. Structural Equation Modeling, 6, 1-55. https://doi. org/10.1080/10705519909540118

45. Jöreskog, K. G. (1993). Testing structural equation models. Sage focus editions, 154, 294-294.

46. Putnick, D. L., \& Bornstein, M. H. (2016). Measurement invariance conventions and reporting: The state of the art and future directions for psychological research. Developmental Review, 41, 71-90. https://doi.org/10.1016/j.dr.2016.06.004

47. Van De Schoot, R., Schmidt, P., De Beuckelaer, A., Lek, K., \& Zondervan-Zwijnenburg, M. (2015). Editorial: Measurement Invariance. Frontiers in Psychology. https://doi.org/10.3389/ fpsyg.2015.01064
48. Svetina, D., Rutkowski, L., \& Rutkowski, D. (2020). Multiplegroup invariance with categorical outcomes using updated guidelines: An illustration using Mplus and the lavaan/semTools packages. Structural Equation Modeling, 27, 111-130. https://doi.org/ 10.1080/10705511.2019.1602776

49. Bovaird, J.A., Koziol, N.A. (2012). Measurement models for ordered-categorical indicators. In Handbook of structural equation modeling. The Guilford Press pp. 495-511.

50. Sass, D. A., Schmitt, T. A., \& Marsh, H. W. (2014). Evaluating model fit with ordered categorical data within a measurement invariance framework: A comparison of estimators. Structural Equation Modeling, 21, 167-180. https://doi.org/10.1080/10705 511.2014.882658

51. Cheung, G. W., \& Rensvold, R. B. (2002). Evaluating goodnessof-fit indexes for testing measurement invariance. Structural Equation Modeling, 9, 233-255. https://doi.org/10.1207/S1532 8007sem0902_5

52. Huang, P. H. (2017). Asymptotics of AIC, BIC, and RMSEA for model selection in structural equation modeling. Psychometrika, 82, 407-426. https://doi.org/10.1007/s11336-017-9572-y

53. Dimitrov, D. M. (2006). Comparing groups on latent variables: A structural equation modeling approach. Work, 26, 429-436.

54. Alavi, M., Visentin, D. C., Thapa, D. K., Hunt, G. E., Watson, R., \& Cleary, M. (2020). Chi-square for model fit in confirmatory factor analysis. Journal of Advanced Nursing, 76, 2209-2211. https://doi.org/10.1111/jan.14399

55. Sawyer, M. G., Arney, F. M., Baghurst, P. A., Clark, J. J., Graetz, B. W., Kosky, R. J., Nurcombe, B., Patton, G. C., Prior, M. R., \& Raphael, B. (2001). The mental health of young people in Australia: Key findings from the child and adolescent component of the national survey of mental health and well-being. Australian \& New Zealand Journal of Psychiatry, 35, 806-814.

56. Hughes, K., Bellis, M. A., Hardcastle, K. A., Sethi, D., Butchart, A., Mikton, C., Jones, L., \& Dunne, M. P. (2017). The effect of multiple adverse childhood experiences on health: A systematic review and meta-analysis. Lancet Public Health, 2, E356-E366.

57. Ashton, K., Bellis, M., \& Hughes, K. (2016). Adverse childhood experiences and their association with health-harming behaviours and mental wellbeing in the Welsh adult population: A national cross-sectional survey. Lancet, 388, 21-21.

58. Felitti, V. J., Anda, R. F., Nordenberg, D., Williamson, D. F., Spitz, A. M., Edwards, V., Koss, M. P., \& Marks, J. S. (1998). Relationship of childhood abuse and household dysfunction to many of the leading causes of death in adults: The adverse childhood experiences (ACE) study. American Journal of Preventive Medicine, 14, 245-258. https://doi.org/10.1016/S0749-3797(98) 00017-8

59. Nelson, C. A., Bhutta, Z. A., Burke Harris, N., Danese, A., \& Samara, M. (2020). Adversity in childhood is linked to mental and physical health throughout life. BMJ, 371, m3048. https://doi.org/ 10.1136/bmj.m3048

60. Koushede, V., Lasgaard, M., Hinrichsen, C., Meilstrup, C., Nielsen, L., Rayce, S. B., Torres-Sahli, M., Gudmundsdottir, D. G., Stewart-Brown, S., \& Santini, Z. I. (2019). Measuring mental well-being in Denmark: Validation of the original and short version of the Warwick-Edinburgh mental well-being scale (WEMWBS and SWEMWBS) and cross-cultural comparison across four European settings. Psychiatry Research, 271, 502-509. https://doi. org/10.1016/j.psychres.2018.12.003

61. Page, N., Hewitt, G., Young, H., Moore, G., \& Murphy, S. (2021). Student Health and Wellbeing in Wales: Report of the 2019/20 School Health Research Network Student Health and Wellbeing Survey. Cardiff University.

62. Warwick Medical School. WEMWBS in other languages. Available online: https://warwick.ac.uk/fac/sci/med/research/platform/ wemwbs/using/translations. Accessed 13 Oct. 
63. Sebba, J., Berridge, D., Luke, N., Fletcher, J., Bell, K., Strand, S., Thomas, S., Sinclair, I., \& O'Higgins, A. (2015). The educational progress of looked after children in England: Linking care and educational data. University of Oxford Department of Education.

Publisher's Note Springer Nature remains neutral with regard to jurisdictional claims in published maps and institutional affiliations. 